\title{
Development of a Loop-Mediated Isothermal Amplification Procedure as a Sensitive and Rapid Method for Detection of 'Candidatus Liberibacter solanacearum' in Potatoes and Psyllids
}

\author{
Aravind Ravindran, Julien Levy, Elizabeth Pierson, and Dennis C. Gross
}

First and fourth authors: Department of Plant Pathology and Microbiology, and second and third authors: Department of Horticultural Sciences, Texas A\&M University, College Station 77843.

Accepted for publication 12 May 2012.

\begin{abstract}
Ravindran, A., Levy, J., Pierson, E., and Gross, D. C. 2012. Development of a loop-mediated isothermal amplification procedure as a sensitive and rapid method for detection of 'Candidatus Liberibacter solanacearum' in potatoes and psyllids. Phytopathology 102:899-907.

This study reports the development of a loop-mediated isothermal amplification procedure (LAMP) for polymerase chain reaction (PCR)based detection of 'Candidatus Liberibacter solanacearum', the bacterial causal agent of potato zebra chip (ZC) disease. The 16S rDNA gene of ' $\mathrm{Ca}$. Liberibacter solanacearum' was used to design a set of six primers for LAMP PCR detection of the bacterial pathogen in potato plants and

the psyllid vector. The advantage of the LAMP method is that it does not require a thermocycler for amplification or agarose gel electrophoresis for resolution. Positive LAMP results can be visualized directly as a precipitate. The LAMP strategy reported here reliably detected ' $\mathrm{Ca}$. Liberibacter solanacearum' and the closely related species ' $\mathrm{Ca}$. Liberibacter asiaticus', the causative agent of huanglongbing disease of citrus, in plant DNA extracts. Although not as sensitive as quantitative real-time PCR, LAMP detection was equivalent to conventional PCR in tests of ZC-infected potato plants from the field. Thus, the LAMP method shows strong promise as a reliable, rapid, and cost-effective method of detecting ' $\mathrm{Ca}$. Liberibacter' pathogens in psyllids and field-grown potato plants and tubers.
\end{abstract}

Zebra chip (ZC) disease of potatoes has caused important economic losses in the United States, Mexico, and New Zealand (26). In the United States, the disease was observed first in Texas in 2000, and is spreading to other potato-growing regions of the United States $(1,3,26)$. ZC is caused by a phloem-limited bacterium identified as 'Candidatus Liberibacter solanacearum', which is transmitted by the potato psyllid Bactericera cockerelli (17). Symptoms of ' $C a$. Liberibacter solanacearum'-infected potatoes include curling, purpling or chlorosis of leaves, and stunted plants with swollen internodes or aerial tubers $(26,33)$. Tuber symptoms include darkening of the medullary rays. This tuber symptom is especially problematic for the potato chip industry because it is intensified with frying, making chips unsuitable for marketing. The discoloration of chips led to the name zebra chip (ZC) for the disease. The disease also causes reduced yield and premature plant death, thus impacting fresh market production too. A closely related ' $\mathrm{Ca}$. Liberibacter' species, ' $\mathrm{Ca}$. Liberibacter asiaticus', causes huanglongbing disease (HLB, also referred to as citrus greening) in citrus $(7,9,29)$. Similar to ' $C a$. Liberibacter solanacearum', ' $C a$. Liberibacter asiaticus' is a noncultivable, phloem-limited pathogen and is transmitted by the Asian citrus psyllid, Diaphorina citri. Although plant diseases caused by psyllid transmission of ' $\mathrm{Ca}$. Liberibacter' did not occur in the United States prior to 2000, they are now threatening both potato and citrus production $(13,33)$.

There are no effective control strategies for protecting plants from ' $\mathrm{Ca}$. Liberibacter' species. Existing measures depend largely on the early detection and control of the psyllid vectors as well as

Corresponding author: D. C. Gross; E-mail address: d-gross@tamu.edu

*The $e$-Xtra logo stands for "electronic extra" and indicates that Figures 1 and 4 appear in color online.

http://dx.doi.org/10.1094/PHYTO-03-12-0055-R

(c) 2012 The American Phytopathological Society early detection of ' $\mathrm{Ca}$. Liberibacter' in the vector and field plants (5). Accurate, affordable, and accessible pathogen detection is essential for timely disease control and for reducing management costs. Current detection methods for ' $\mathrm{Ca}$. Liberibacter solanacearum' in insects and plants rely primarily on conventional polymerase chain reaction (PCR) assays, although methods utilizing quantitative real-time PCR (qPCR) also have been developed $(12,14)$. Conventional PCR protocols are based on the use of primers designed for the $16 \mathrm{~S}$ rDNA sequence, 16-23S rDNA intergenic region, and the housekeeping gene, adenylate kinase $(a d k)$ of ' $C a$. Liberibacter solanacearum' $(2,5,14,22,26,33)$. For qPCR, the 16S rDNA specific primers, LsoF and HLBr, are used almost exclusively (15). Despite progress in optimizing these methods for increased reliability, they are expensive (e.g., require thermal cycling equipment), time consuming (e.g., require postPCR analysis via gel electrophoresis or qPCR software), and must be conducted in a laboratory environment.

A relatively new technology for bacterial pathogen detection known as loop-mediated isothermal amplification (LAMP), developed by Notomi et al. (20), has the advantages of being highly specific, rapid, efficient, and laborsaving. In contrast to both conventional PCR and qPCR, a precision thermocycler is not needed because LAMP is performed at a single temperature. To date, LAMP has found success in medical laboratories for the rapid detection of bacteria in specimens from humans (8), and has been used for detection of a few vascular plant pathogens including Erwinia amylovora (30), Ralstonia solanacearum (11), and Phytophthora spp. (32). Recently, LAMP was developed for the detection of ' $\mathrm{Ca}$. Liberibacter asiaticus' using a set of four primers designed for nucleotide sequences located either in the $16 \mathrm{~S}$ rDNA gene or within the nusG-rplKAJL-rpoB gene sequence (21). No such methods currently exist for diagnosis of ' $\mathrm{Ca}$. Liberibacter solanacearum'.

LAMP as originated by Notomi et al. (20) is a nonquantitative PCR method based on autocycling strand displacement DNA 
synthesis facilitated by a Bacillus stearothermophilus (Bst) DNA polymerase. However, unlike conventional PCR, LAMP is a onestep process that amplifies a target DNA sequence at a single temperature using sets of six primers, including two inner primers (FIP and BIP), two outer primers (F3 and B3), and two loop primers (LF and LB) that are specific for eight independent regions of the target sequence (Fig. 1). The forward inner primer (FIP) anneals to initiate the first-strand synthesis of the target sequence by the Bst polymerase. The forward outer primer (F3, at a lower concentration) then hybridizes and displaces the synthesized first strand. Unlike the template, the newly synthesized first strand incudes additional sequence corresponding to the FIP primer at the $5^{\prime}$ end. The FIP primer contains a sequence complementary to the F1 region of the target strand, which facilitates the formation of a loop structure. Similarly the backward inner primer (BIP, containing a copy of the complementary sequence of the $\mathrm{B} 1$ region of the target) anneals to the newly synthesized first strand to create a final template with sequences that are complementary to F1 at one end and B1 at the other. These complementary regions fold back and anneal to the $\mathrm{F} 1$ and $\mathrm{B} 1$ regions of the target sequence to form a dumbbell shape (Fig. 1). The dumbbellshaped, stem-loop structure acts as a template for the synthesis of long concatemeric strands consisting of repeating units of the target sequence (20). Furthermore, an accelerated LAMP procedure has been developed that employs two additional loop primers (LF and LB) for enhanced specificity and reaction efficiency (19).

LAMP amplification has the potential for increased detection specificity in comparison to conventional PCR methods that are reliant on the sequence specificity of two primers and two annealing regions. In contrast, LAMP is dependent on the formation of dumbbell-shaped final templates for amplification, which in turn are reliant on the sequence specificity of six primers and eight annealing regions. When there is amplification, the LAMP method produces substantial amounts of large DNA strands composed of

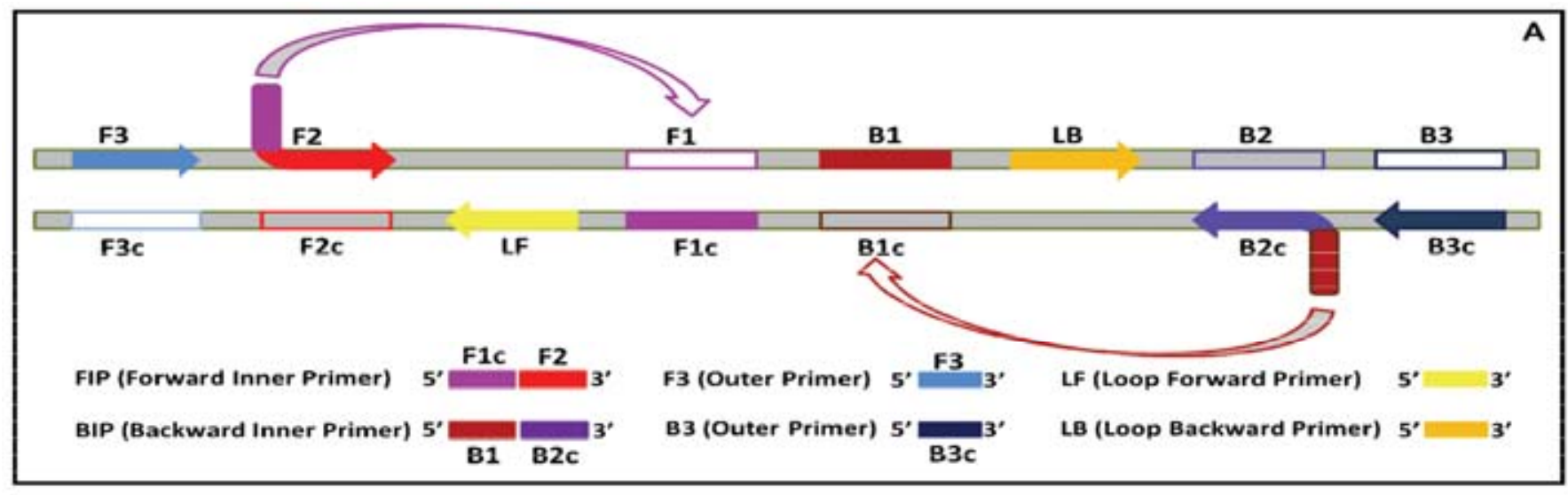

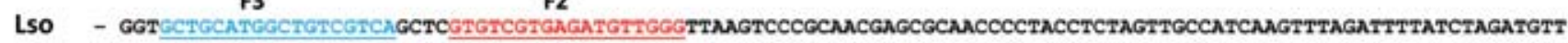
LSO-C - cCacGacGtaccGacagcagtcgagcacagcactctacaAccca CGTTGGGGATGGAGATCAACGGTAGTTCAAATCTAAAATAGATCTACAA F1c

Lso - GGGTACIITATAGGGACTGCCGGTGATMATCC LB

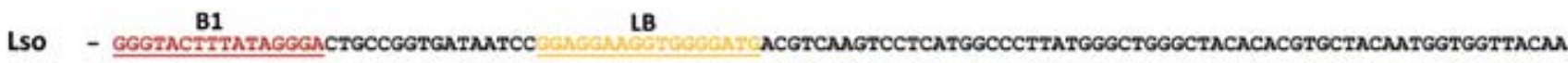
LSO-C - CCCATGAAATATCCCTGACGGCCACTATTAGGCCTCCTTCCACCCCTACTGCAGTTCAGGAGTACCGGGAATACCCGACCCGATGTGTGCACGATGTTACCACCAATGTT $\mathrm{B2C}$

\begin{tabular}{|l|l|}
\hline \multicolumn{2}{|c|}{ C } \\
\hline LAMP Primers & $5^{\prime}$-3' \\
\hline LsoTX16SLamp-F3 & CTGCATGGCTGTCGTCA \\
\hline LsoTX16SLamp-B3 & GTAACCACCATTGTAGC \\
\hline LsoTX16SLamp-FIP (F1C+F2) & TAGAGGTAGGGGTTGC-GTGTCGTGAGATGTTGGG \\
\hline LsoTX16SLamp-BIP (B1+B2C) & GGGTACTTTATAGGGA-CCATGAGGACTTGACG \\
\hline LsoTX16SLamp-LF & GCTCGTTGCGGGACTTA \\
\hline LsoTX16SLamp-LB & GGAGGAAGGTGGGGATG \\
\hline
\end{tabular}

Fig. 1. Structure and sequence of the loop-mediated isothermal amplification procedure (LAMP) primers. A, Schematic diagram of the 16S rDNA target showing the location of the eight annealing regions for the six LAMP primers. The forward inner primer (FIP) consisting of F1c (the complementary sequence of the F1 region) and the F2 region will bind first to the F2 region and then F1c forming the forward stem loop structure. The backward inner primer (BIP) consisting of B1 and the B2c (the complementary sequence of the B2 region) will bind first to the B2c region and then B1 forming the backward stem loop structure. The outer primers F3 and B3 (required for initiation of the LAMP reaction) will bind to the F3 and B3 regions, respectively. The loop primers LF and LB (used to accelerate the amplification process) will bind the LF (between the F2 and F1c binding regions) and LB (between the B2c and B1 binding regions), respectively. B, Partial nucleotide sequence of the 16S rDNA gene from 'Candidatus Liberibacter solanacearum' (GenBank accession no. NC_014774). The sequences of primers FIP (F1c-F2), BIP (B1-B2c), F3, B3, LF, and LB are shown in varying shades of gray. C, The primer sequence for each of the six LAMP primers. 
concatemers of the target sequence. A by-product of the LAMP amplification is the formation of an insoluble white precipitate of magnesium pyrophosphate, which becomes increasingly visible with time in the reaction tube. The results (no product versus visible turbidity) make it simple to distinguish between negative and positive reactions within the reaction tube. The double-stranded, LAMP-amplified concatemers of the target DNA produce a characteristic ladder banding pattern defined by the number of target sequence copies when analyzed via gel electrophoresis. LAMP amplification requires less than $60 \mathrm{~min}$ (as compared to up to $2 \mathrm{~h}$ for conventional PCR amplification) and no post-PCR analysis via gel electrophoresis is needed to visualize the outcome.

This report describes the design of a six-primer set and the optimization of a protocol for LAMP detection of ' $\mathrm{Ca}$. Liberibacter solanacearum' in plant and insect samples. A number of ' $\mathrm{Ca}$. Liberibacter solanacearum' genes were analyzed to determine whether the required six LAMP primers could be designed to amplify ' $C a$. Liberibacter solanacearum' gene-specific target sequences. Although a number of gene-specific LAMP primer sets were designed and tested, only primers targeting the $16 \mathrm{~S}$ rDNA region provided reproducible results. Using this $16 \mathrm{~S}$ rDNA LAMP primer set (hereafter LsoTX16SLAMP), the optimal LAMP conditions were determined. Previously, a common problem with LAMP was reproducibility $(10,31)$, and this report provides a reliable and reproducible LAMP method for the detection of ' $\mathrm{Ca}$. Liberibacter solanacearum'. Data on the specificity and sensitivity of the optimized protocol are presented. A comparison of the capability of conventional PCR, qPCR, and LAMP to detect ' $\mathrm{Ca}$. Liberibacter solanacearum' in plant samples is provided. Several different devices ranging from a thermocycler to less expensive water baths and heating blocks were tested for their ability to maintain the correct isothermal conditions for LAMP amplification. The goal was to develop a rapid and robust method for the detection of ' $\mathrm{Ca}$. Liberibacter solanacearum' for use in less well-equipped laboratories and potentially at field sites.

\section{MATERIALS AND METHODS}

Plant and insect material. Healthy and ZC-symptomatic potato plants and tubers were collected from commercial fields in Texas, Kansas, and Nebraska. In the case of potato tubers, ZC symptoms were based on the results of the fry test, e.g., darkening of the medullary rays when tubers were chipped and fried (17). Also included in the study were glasshouse-grown plants that were either exposed to or protected from bacterialiferous psyllids. The protected glasshouse plants served as ' $\mathrm{Ca}$. Liberibacter solanacearum'-negative controls, whereas the psyllid-infested plants served as ' $C a$. Liberibacter solanacearum'-positive controls. ' $\mathrm{Ca}$. Liberibacter solanacearum'-infected insects were obtained from laboratory populations described previously (18). DNA samples from citrus plants growing in Florida that were symptomatic for HLB and subsequently shown to have ' $\mathrm{Ca}$. Liberibacter asiaticus' via conventional PCR were provided by Nian Wang, University of Florida, and used in the LAMP specificity assays.

Bacterial strains. Strains of bacteria within the Rhizobiaceae were used in tests of specificity of the LAMP primers designed for ' $C a$. Liberibacter solanacearum' detection. The bacteria and strains are as follows: Agrobacterium tumefaciens (strains C58, biovar 1; K84, biovar 2; GV3101), A. vitis (strain A804, biovar 3), A. rhizogenes (strain A4, biovar 2), Rhizobium leguminosarum bv. trifolii (strain 162P30), R. leguminosarum (strain 128A12), $R$. leguminosarum bv. phaseoli (strains 3610, and 127k14), and Sinorhizobium meliloti (strain 102F28).

Extraction of DNA. Plant DNA was extracted from different plant tissues including tubers, roots, stems, petioles, and leaves of potato. Total DNA (plant and bacteria) was extracted from the different plant tissues using a method modified from Doyle and Doyle (4), as described previously (22). Total DNA (insect and bacteria) was extracted from pooled samples of 10 adult psyllids, as described previously (18).

Primer design for LAMP. The structure and sequence of the six primers and their annealing sites within the 16S rDNA target sequence are given in Figure 1. These include forward and backward external primers F3 and B3, respectively; forward and backward internal primers FIP and BIP, respectively; and forward and backward loop primers LF and LB, respectively.

Potential target sequences of ' $\mathrm{Ca}$. Liberibacter solanacearum' genes (GenBank accession NC_014774) were selected based on their suitability for designing the six primers sequences (and eight annealing regions) required for LAMP amplification using the primer design software, Primer Explorer V4 software (Eiken Co., Ltd., Japan: http://primerexplorer.jp/e/). Primer Explorer V4 considered potential adverse interactions among the six primers (e.g., whether primer sequences will form hairpins or primer dimers that might result in false positives and whether specific primer combinations will self-hybridize). To verify the primer folding predictions and the free energy of the most stable DNA secondary structure, they were calculated by UNAFold software (16). Six LAMP primers specific to each of several housekeeping genes (i.e., rpoB, rpoD, gyrB, adk, recA, dnaG, fumC, pyrE, kdo, ftsA, gnd, $m d h$, and sucC) along with the $16 \mathrm{~S}$ rDNA gene and the $16 \mathrm{~S} / 23 \mathrm{~S}$ rDNA intergenic region were designed and tested. Primers for all of the genes amplified the ' $\mathrm{Ca}$. Liberibacter solanacearum' target sequence, although somewhat unreliably with either no reproducible amplification or false positives. Optimization of the turn-back primers (FIP and BIP) for each gene sequence was required for stable loop formation and this was carried out using the least absolute shrinkage and selection operator (LASSO) regression analysis method (http://gerg.gsc.riken.jp/ TP_optimization/) (10). Only the resulting 16S rDNA primer set, LsoTX16SLAMP, amplified the ' $C a$. Liberibacter solanacearum' target sequence reliably and this six primer set was used for the development of LAMP detection of ' $\mathrm{Ca}$. Liberibacter solanacearum'.

Standard desalted F3, B3, LF, and LB primers were obtained from either Invitrogen (Grand Island, NY) or IDT (Coralville, IA). High-performance liquid chromatography-purified FIP and BIP primers (Invitrogen) are highly recommended and were used to improve the reproducibility of LAMP amplification.

Optimization of LAMP reaction. Based on the procedure of Tomita et al. (31), a temperature of $60^{\circ} \mathrm{C}$ and a time of $60 \mathrm{~min}$ was used to optimize the reaction components, i.e., the Bst DNA polymerase enzyme (New England BioLabs) concentration (2, 4, and 8 units), the $\mathrm{MgSO}_{4}$ (New England BioLabs) concentration $(2,4,6,8,10,12,14$, and $16 \mathrm{mM})$, the betaine concentration (Sigma-Aldrich) $(0.8,1.2,1.6,2.0$, and $2.4 \mathrm{M})$. Furthermore, the reaction temperature $\left(57,60,63,66\right.$, and $\left.69^{\circ} \mathrm{C}\right)$, and the amplification time $(15,30,45,60$, and $75 \mathrm{~min})$ were optimized as described by Yeh et al. (35). The optimal conditions for LAMP using the LsoTX16SLAMP primers are given in Table 1 and described further in the Results.

LAMP reactions were performed in $25 \mu$ (total volume) in 200- $\mu$ l PCR tubes (Denville Scientific Inc.). For multiple samples, a standard mix containing the ThermoPol Reaction Buffer (New England BioLabs), betaine, $\mathrm{MgSO}_{4}$, dNTPs (Fisher Scientific), and the six primers was prepared according to Table 1. For fluorescent visualization (see below), calcein and $\mathrm{MnCl}_{2}$ were added to the standard mix according to Table 1. The Bst DNA polymerase enzyme was added to the standard mix immediately before a $23-\mu \mathrm{l}$ aliquot of the mix was dispensed to each sample tube, and $2 \mu \mathrm{l}$ of total DNA ( 30 to $50 \mathrm{ng} / \mu \mathrm{l})$ extract from plants was supplied. The sample was mixed only by the careful addition (via pipettor) of the DNA into the mix; more aggressive mixing may inactivate the $B s t$ DNA polymerase. The best amplification occurred at $60^{\circ} \mathrm{C}$ with the amplification time of $60 \mathrm{~min}$ followed by incubation at $80^{\circ} \mathrm{C}$ for $5 \mathrm{~min}$ to terminate the elongation reaction to inactivate the $B s t$ DNA polymerase. 
Direct analysis of LAMP products. LAMP amplicons were detected directly in the reaction tubes by formation of an insoluble magnesium pyrophosphate precipitate during amplification; turbidity indicated a positive reaction, whereas a negative result remained clear. Detection can be enhanced via the addition of $1 \mathrm{mM} \mathrm{MnCl}$ and $50 \mu \mathrm{M}$ calcein (Wako Chemical) to the reaction mix, which results in the formation of a fluorescent green metal indicator (31). A positive reaction was indicated by green fluorescence and a negative reaction by the lack of fluorescence. A UV lamp (wavelength, 254/365 nm) was used to illuminate the fluorescent product for color photography and black and white imaging. Results were further confirmed via gel electrophoresis on a $2 \%$ agarose gel.

Multiple sequence alignment and specificity of LAMP. Partial nucleotide sequences of the $16 \mathrm{~S}$ rDNA gene available in the National Center for Biotechnology Information (NCBI) database from ' $\mathrm{Ca}$. Liberibacter solanacearum' (GenBank accession NC_014774.1) and other closely related members of the Rhizobiaceae including ' $\mathrm{Ca}$. Liberibacter asiaticus' [GenBank accession NC_012985.3], A. tumefaciens [GenBank accession AB247614.1], and S. meliloti [GenBank accession EU182657.1]) were compared with CLUSTAL X Multiple Sequence Alignment (28). The specificity of LAMP using the LsoTX16SLAMP primer set was examined using DNA samples from these bacterial species. The quality of the bacterial DNA was verified by PCR amplification of the 16S rDNA gene using the 16S rDNA universal primers ( $\mathrm{pA}$ and $\mathrm{pH}$ forward and reverse primers, respectively) as described previously $(27,34)$. As controls, DNA was extracted from ' $\mathrm{Ca}$. Liberibacter solanacearum'-uninfected potato and tomato and ' $\mathrm{Ca}$. Liberibacter asiaticus'-uninfected citrus. The quality of plant DNA extracts from potato and tomato was verified by PCR amplification of the $\beta$-tubulin gene, as described previously (22).

Sensitivity of LAMP and comparison to conventional and qPCR. To test the sensitivity of the LsoTX16SLAMP primer set, LAMP was performed on a dilution series of DNA extracted from a ZC-symptomatic potato plant. The DNA sample was diluted 10 -fold from $10^{2}$ to $10^{-5} \mathrm{ng}$ and the presence of a LAMP product was determined via direct visualization and electrophoresis on a $2 \%$ agarose gel. For comparison, conventional PCR using the LsoTX16/23 F/R primers for detection of ' $C a$. Liberibacter solanacearum' was performed as described previously (22).

LAMP, conventional PCR, and qPCR methods were compared using DNA samples extracted from four different plant tissues (tuber, leaf, stem, and petiole) from 10 different ZC-symptomatic plants collected from a field in Weslaco, TX. As above, the LsoTX16/23 F/R primers were used for the conventional PCR.
For qPCR, the LsoF and HLBr primers and SYBR green methodology were used as described previously (12).

LAMP in different isothermal reactors. The LAMP method was optimized using a thermocycler (ABI Geneamp 9700 thermocycler, Applied Biosystems, Foster City, CA) to maintain a constant reaction temperature. Different types of isothermal reactors, including a heating block (MyBlock Mini dry bath, Benchmark Scientific), incubator (Economy, Precision), and water bath (180 series, Precision) were evaluated to determine whether they could be used to incubate tubes at a sufficiently stable temperature for LAMP amplification.

\section{RESULTS}

LAMP primer design. Several genes were selected as potential target sequences for LAMP primer design, including the $16 \mathrm{~S}$ rDNA gene, 16S/23S rDNA intergenic region, and several housekeeping genes (i.e., rpoB, rpoD, gyrB, adk, recA, dnaG, fumC, pyrE, kdo, ftsA, gnd, mdh, and sucC) using primer design software (Primer Explorer V4). Except for the 16S rDNA sequence and the $16 / 23 \mathrm{~S}$ rDNA intergenic region, all of the ' $\mathrm{C} a$. Liberibacter solanacearum' genes evaluated had between 33 to $41 \%$ GC content, making them unsuitable for LAMP. Analysis of the ' $\mathrm{Ca}$. Liberibacter solanacearum' genome revealed that only the three $16 / 23 \mathrm{~S}$ rDNA gene copies had GC content higher than $45 \%$. Despite the low GC content of the housekeeping genes evaluated, the primer design software identified a target sequence within each of these genes that was acceptable for designing all six LAMP primers. However, using these gene-specific primer sets, LAMP failed to amplify a target sequence for any of the genes including the $16 \mathrm{~S}$ rDNA sequence and the $16 / 23 \mathrm{~S}$ rDNA intergenic region (data not shown).

In addition to consideration of annealing factors and GC content, the design of the forward inner primer and the backward inner primer (the turn-back primers) were found previously to be the most important feature for reproducible amplification (10). The application of both the UNAFold (16) and LASSO regression analysis (http://gerg.gsc.riken.jp/TP_optimization/) software program to design primers was shown (10) to be the best method for designing the turn-back primers. Using this method improved LAMP primers for all of the genes were designed. Only the LAMP primers specific for the ' $C a$. Liberibacter solanacearum' $16 \mathrm{~S}$ rDNA reproducibly amplified the ' $\mathrm{Ca}$. Liberibacter solanacearum' target sequence, and these were used for further study.

Optimal conditions for detection of ' $\mathrm{Ca}$. Liberibacter solanacearum' using LAMP. Optimal reaction conditions are found in Table 1. The optimization of the LAMP protocol was

TABLE 1. Composition of the loop-mediated isothermal amplification procedure (LAMP) reaction mixture for detection of 'Candidatus Liberibacter solanacearum' and 'Ca. Liberibacter asiaticus'

\begin{tabular}{|c|c|c|c|c|}
\hline Components & Stock concentration & Working concentration & Required concentration & Required volume/reaction \\
\hline Double distilled water (RNAse free water) & $6.20 \mu \mathrm{l}$ & & & \\
\hline ThermoPol reaction buffer ${ }^{\mathrm{a}}$ & $10 x$ & $10 x$ & $2 \times$ & $5.00 \mu \mathrm{l}$ \\
\hline Betaine & $5 \mathrm{M}$ & $5 \mathrm{M}$ & $1.6 \mathrm{M}$ & $4.00 \mu \mathrm{l}$ \\
\hline dNTPs & $25 \mathrm{mM}$ & $25 \mathrm{mM}$ & $2.8 \mathrm{mM}$ & $1.40 \mu \mathrm{l}$ \\
\hline F3 primer & $100 \mathrm{pM}$ & $100 \mathrm{pM}$ & $10 \mathrm{pM}$ & $0.10 \mu \mathrm{l}$ \\
\hline B3 primer & $100 \mathrm{pM}$ & $100 \mathrm{pM}$ & $10 \mathrm{pM}$ & $0.10 \mu \mathrm{l}$ \\
\hline FIP & $100 \mathrm{pM}$ & $100 \mathrm{pM}$ & $40 \mathrm{pM}$ & $0.20 \mu \mathrm{l}$ \\
\hline LB & $100 \mathrm{pM}$ & $100 \mathrm{pM}$ & $20 \mathrm{pM}$ & $0.40 \mu \mathrm{l}$ \\
\hline $\mathrm{MnCl}_{2}$ & $20 \mathrm{mM}$ & $20 \mathrm{mM}$ & $1 \mathrm{mM}$ & $1.25 \mu \mathrm{l}$ \\
\hline Calcein & $1 \mathrm{mM}$ & $1 \mathrm{mM}$ & $50 \mu \mathrm{M}$ & $1.25 \mu \mathrm{l}$ \\
\hline 8 units of Bst DNA polymerase ${ }^{\mathrm{b}}$ & & & & $1.00 \mu \mathrm{l}$ \\
\hline Template DNA $(\approx 50 \mathrm{ng} / \mu \mathrm{l})$ & & & & $2.00 \mu \mathrm{l}$ \\
\hline Total reaction volume & & & & $25.00 \mu \mathrm{l}$ \\
\hline
\end{tabular}

a Purchased from New England BioLabs.

${ }^{\mathrm{b}}$ Bacillus stearothermophilus DNA polymerase. 
conducted using DNA extracted from ' $\mathrm{Ca}$. Liberibacter solanacearum'-infected plant material as the positive control and DNA extracted from a healthy plant as the negative control. LAMP primers were added in concentrations as recommended previously (31). Accordingly, the F3 and B3 primers, required only for the initial displacement reaction, were added at a lower (fourfold) concentration than the FIP and BIP primers. The LF and LB primers are not required for the generation of the template or subsequent amplification, and theoretically can be omitted from the reaction (19). However, when they were added at the recommended concentration (e.g., twofold lower than the FIP and BIP primers), they greatly increased the speed of the reaction by providing an increased number of starting points for DNA synthesis, as observed previously (19). The optimal concentration of Bst polymerase, $\mathrm{MgSO}_{4}$, and betaine were found to be, respectively, 8 units per reaction, $12 \mathrm{mM}$, and $1.6 \mathrm{M}$. LAMP amplification was evaluated at temperatures ranging from 57 to $69^{\circ} \mathrm{C}$ (Fig. 2A). Evaluation of amplification time showed a LAMP product initially being formed after $45 \mathrm{~min}$ (Fig. 2B). No amplification was observed for the water control (Fig. 2) and the healthy plant DNA (data not shown). Thus, the optimal amplification temperature and time were $60^{\circ} \mathrm{C}$ and $60 \mathrm{~min}$, respectively.

Visual detection of LAMP products. Several methods for visual detection of LAMP products were evaluated. These included direct observation of solution turbidity in the reaction tube and under UV light, visualization of the formation of the green fluorescent metal indicator directly and under UV illumination, and gel electrophoresis (Fig. 3). The formation of magnesium pyrophosphate precipitate during the synthesis of DNA facilitated detection of positive amplification directly in the reaction tubes (Fig. 3). No detectable level of precipitate formed in the negative reactions and the solution appeared clear. The precipitate also was clearly visible under UV light, where it appeared as a bright white image with a gel imaging system (Fig. 3). As observed previously (31), addition of $\mathrm{MnCl}_{2}$ and calcein produced a green fluorescence during DNA synthesis, which was more apparent under UV light (Fig. 3). The fluorescent emission is produced as quenching is relieved by manganese chelated to the calcein and pyrophosphate scavenging of the manganese. This emission is further intensified as magnesium binds with the manganese-free calcein. When resolved via gel electrophoresis, LAMP reactions produce many
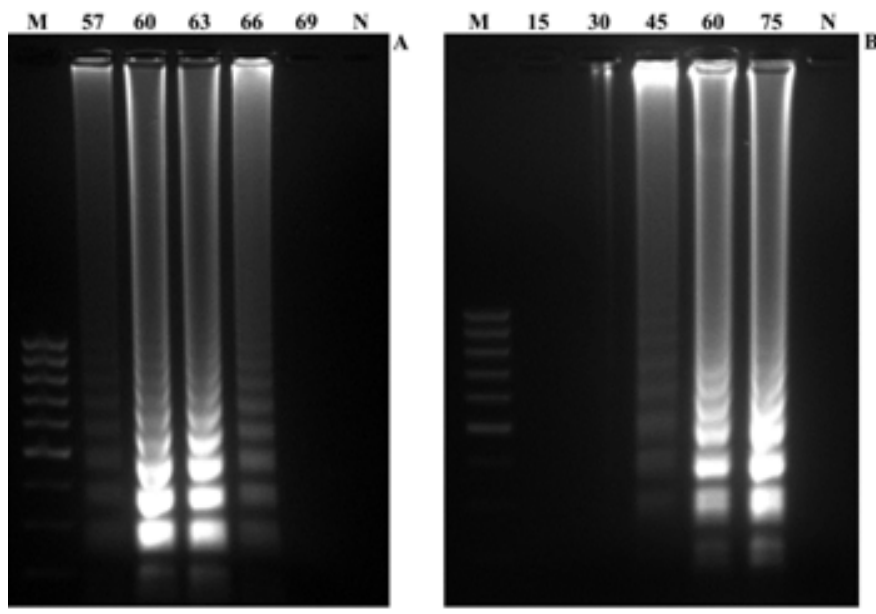

Fig. 2. Optimization of loop-mediated isothermal amplification procedure (LAMP) conditions: effect of amplification temperature and time on amplification of LAMP product. A, Temperature, M, molecular size marker (100-bp DNA ladder, ranging from 100 to $1,000 \mathrm{bp}$ ); LAMP carried out at 57, 60, 63, 66 , and $69^{\circ} \mathrm{C}$, respectively; $\mathrm{N}$, negative control (no DNA). B, Time, M, molecular marker (100-bp DNA ladder, ranging from 100 to 1,000 bp); LAMP carried out for $15,30,45,60$, and 75 min, respectively; $\mathrm{N}$, negative control (no DNA). All the products were examined on $2 \%$ agarose gels and stained with ethidium bromide. different-sized bands in a reproducible pattern (Fig. 3). DNA extracts from psyllid samples and different plant tissues containing ' $C a$. Liberibacter solanacearum' produced similar turbidity, fluorescence, and banding patterns. Negative controls (e.g., no DNA and DNA extracted from healthy plants) produced no turbidity, fluorescence, or DNA bands on gels (Fig. 3).

Specificity of LAMP. The specificity of LAMP using the LsoTX16SLAMP primer set to amplify only ' $C a$. Liberibacter'
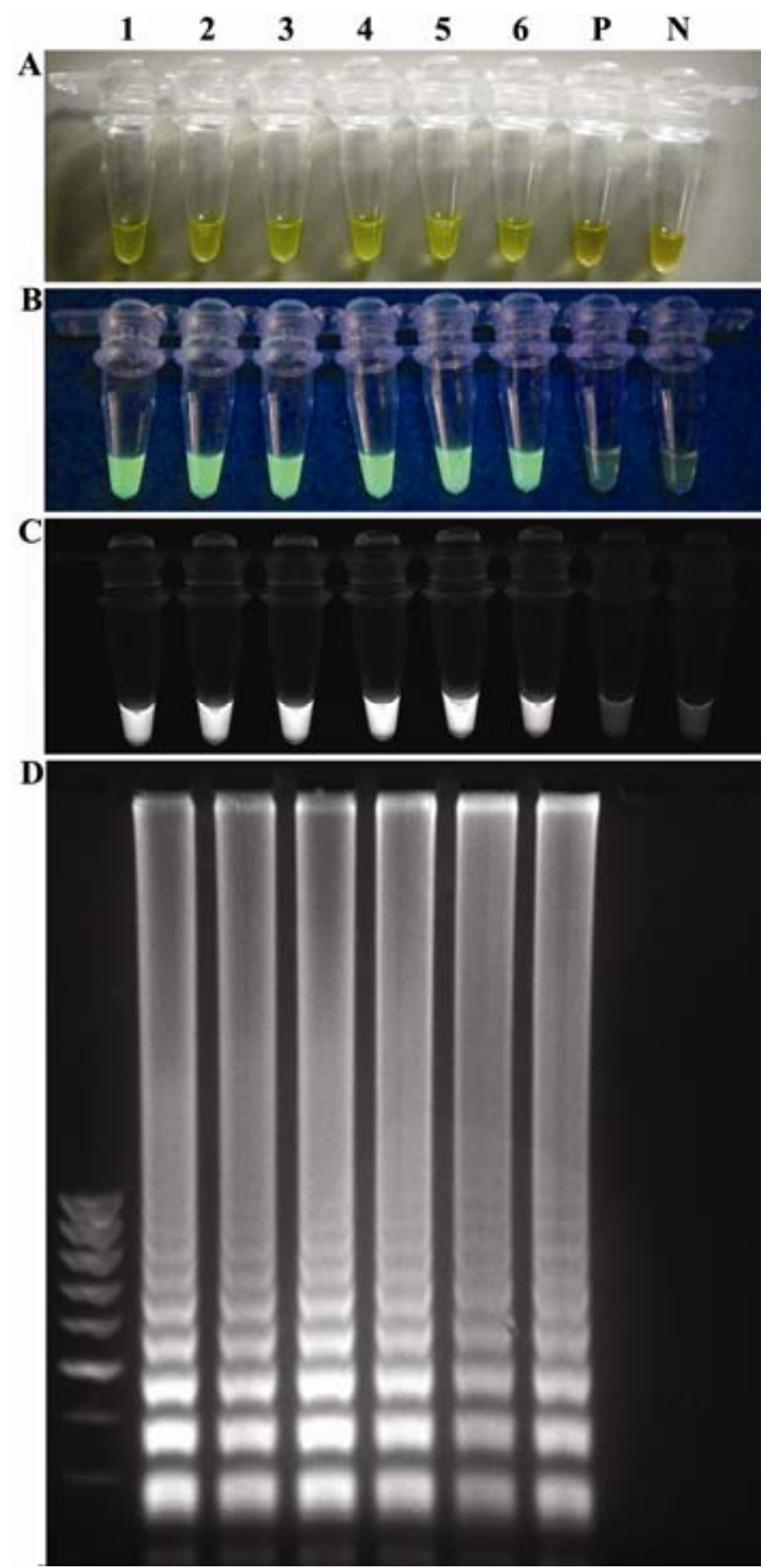

Fig. 3. Loop-mediated isothermal amplification procedure (LAMP) amplification and detection of 'Candidatus Liberibacter solanacearum' using the LsoTX16SLAMP primer set in ' $\mathrm{Ca}$. Liberibacter solanacearum'-infected glasshouse plant tissues and insect samples. Lane 1, tuber; 2, root; 3, stem; 4, petiole; 5, leaf; 6, psyllid; P, plant control (tuber from healthy plant); and $\mathrm{N}$, negative control (no DNA). Visualization A, under normal light, B, under UV light (color image), C, under UV light (black and white image), and D, with ethidium bromide staining on a $2 \%$ agarose gel; a 100-bp DNA ladder appears in the first lane. 
species (' $\mathrm{Ca}$. Liberibacter solanacearum' and ' $\mathrm{Ca}$. Liberibacter asiaticus') was tested using DNA samples from closely related bacterial species including A. tumefaciens, A. vitis, A. rhizogenes, $R$. leguminosarum, and $S$. meliloti (data not shown). The multiple alignment of the partial 16S rDNA nucleotide sequences corresponding to the region used for selection of the LsoTX16SLAMP primers indicated that there was only one nucleotide difference between the ' $C a$. Liberibacter solanacearum' and ' $C a$. Liberibacter asiaticus' sequences, whereas the ' $\mathrm{Ca}$. Liberibacter solanacearum' sequence was only $\approx 85 \%$ similar to the other Rhizobiaceae members tested (Fig. 4A). As expected based on the multiple alignments, the LsoTX16SLAMP primer set amplified products from the ' $C a$. Liberibacter solanacearum' and ' $C a$. Liberibacter asiaticus' samples, but not from A. tumefaciens strain GV3101, S. meliloti strain 102F28, or host plant samples (Fig. 4B and C). Conventional PCR using the bacterial 16S rDNA universal primers and the plant $\beta$-tubulin primers amplified products in all bacterial and plant samples, respectively, indicating the quality of DNA in all samples was equivalent. These experi-
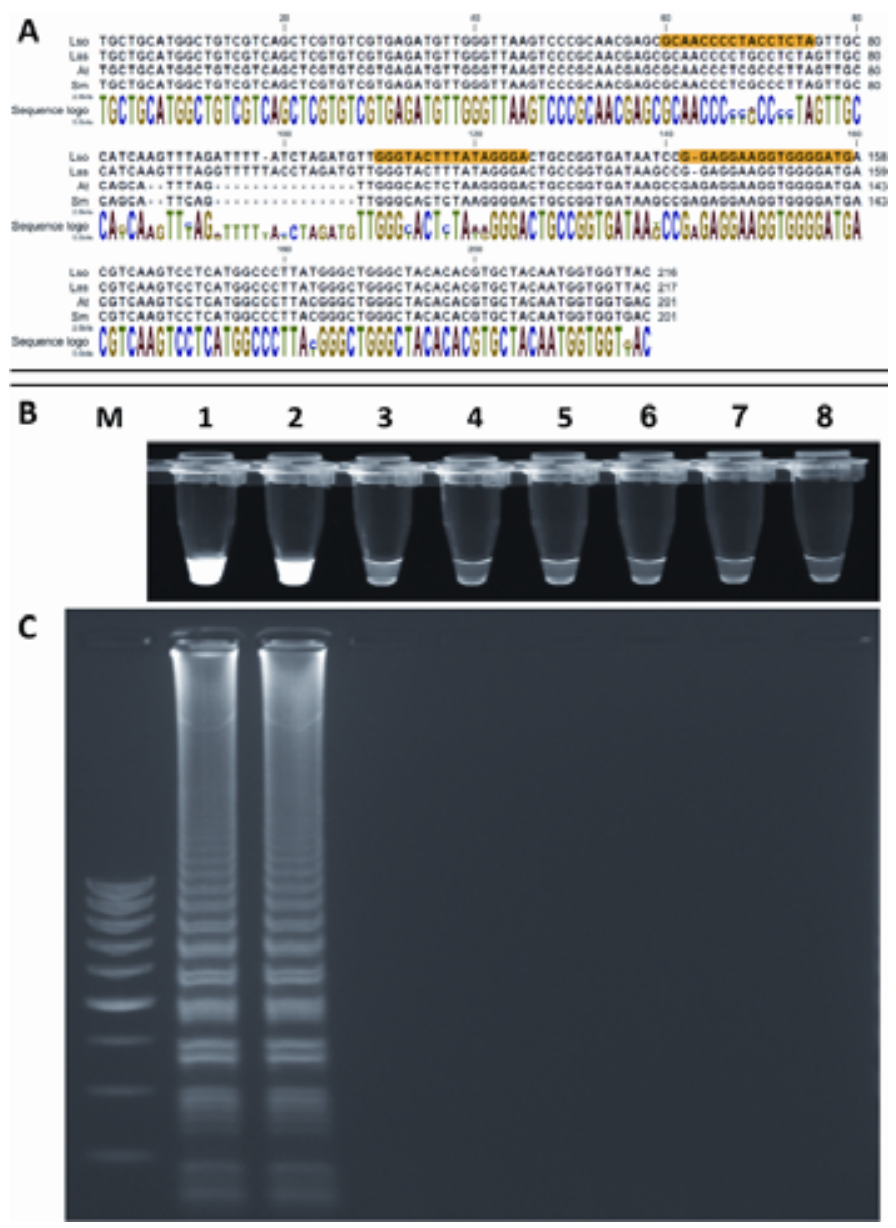

Fig. 4. Loop-mediated isothermal amplification procedure (LAMP) specificity was examined using the LsoTX16SLAMP primer set on DNA samples from closely related bacterial species. A, Multiple alignment of the partial (primer region) $16 \mathrm{~S}$ rDNA nucleotide sequence for 'Candidatus Liberibacter solanacearum' (Lso), 'Ca. Liberibacter asiaticus' (Las), Agrobacterium tumefaciens (At), and Sinorhizobium meliloti (Sm). The five boxed regions correspond to the primer sequence conserved in all four strains; the three highlighted regions in ' $\mathrm{Ca}$. Liberibacter solanacearum' are primer sequences used for specific detection of ' $C a$. Liberibacter' species but not strains of A. tumefaciens and $S$. meliloti. B, Visualization of LAMP product in amplification tube under UV light (black and white image), and $\mathbf{C}$, by gel electrophoresis ( $2 \%$ agarose gel). Lane 1, 'Ca. Liberibacter solanacearum' (positive control); 2, 'Ca. Liberibacter asiaticus' (Las); 3, A. tumefaciens; and 4, S. meliloti. Controls include lane 5, DNA from ' $\mathrm{Ca}$. Liberibacter solanacearum'-uninfected potato; 6, ' $\mathrm{Ca}$. Liberibacter solanacearum'-uninfected tomato; 7, ' $\mathrm{Ca}$. Liberibacter asiaticus'uninfected citrus; and 8, no DNA. ments demonstrated that the primers are specific for the ' $\mathrm{Ca}$. Liberibacter' species we tested (' $\mathrm{Ca}$. Liberibacter solanacearum' and ' $\mathrm{Ca}$. Liberibacter asiaticus') and do not amplify host plant DNA.

Sensitivity of LAMP. The sensitivity of LAMP and conventional PCR to detect ' $C a$. Liberibacter solanacearum' was compared using a 10-fold dilution series from $10^{2}$ to $10^{-5} \mathrm{ng}$ of DNA extracted from a ' $\mathrm{Ca}$. Liberibacter solanacearum'-infected potato tuber. For conventional PCR, bands were clearly visible for samples containing 100 to $1 \mathrm{ng}$ of DNA, whereas LAMP bands were visible for samples containing 100 to $0.01 \mathrm{ng}$ of DNA (Fig. 5). These data suggest that LAMP has the potential to be $\approx 100$ times more sensitive than conventional PCR using the LsoTX16/23 F/R primers.

Comparison of LAMP and conventional PCR results from field samples. Once the protocol was optimized, LAMP and conventional PCR were compared for their ability to detect ' $\mathrm{Ca}$. Liberibacter solanacearum' in field samples from Texas, Kansas, and Nebraska. The DNA was extracted from several plant tissues (tuber, leaf, stem, and petiole); results are shown in Table 2 for samples from 10 different ZC-symptomatic plants collected from a field in Weslaco, TX. The quality and quantity of DNA in all samples was verified by amplification of the plant $\beta$-tubulin

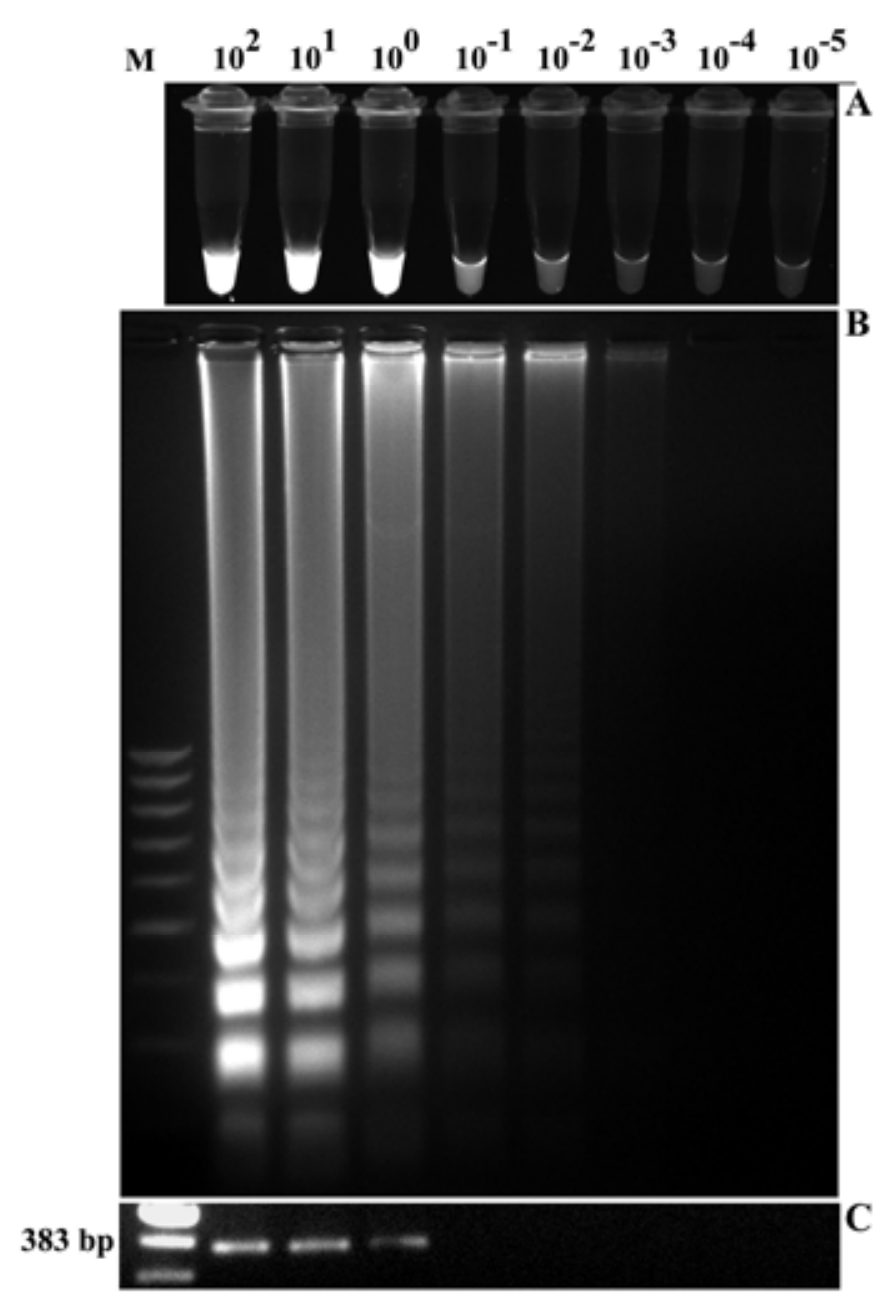

Fig. 5. Sensitivity of detection by loop-mediated isothermal amplification procedure (LAMP) and conventional polymerase chain reaction (PCR): visualization A, under UV light (black and white image), B, LAMP agarose gel, and $\mathbf{C}$, conventional PCR agarose gel. The DNA sample used in the assay was extracted from a zebra chip disease-symptomatic potato plant and diluted tenfold from $10^{2}$ to $10^{-5} \mathrm{ng}$. For conventional PCR, bands are clearly visible for samples containing 100 to $1 \mathrm{ng}$ of DNA, whereas for LAMP, bands are visible for samples containing 100 to $0.01 \mathrm{ng}$ of DNA. 
target, prior to analysis. For 8 out of the $10 \mathrm{ZC}$-symptomatic plants, ' $C a$. Liberibacter solanacearum' was detected in all plant tissues using both techniques (data not shown). Interestingly, all tissues from ZC-symptomatic plant 1 were negative either by LAMP or conventional PCR (Table 2). In addition, the leaf sample from ZC-symptomatic plant 3 was negative for both LAMP and conventional PCR, but the samples from the other tissues (tuber and stem) were positive for both PCR methods. These data demonstrated that the two techniques provided similar diagnostic results. All samples from plants 1 to 10 were then assayed by qPCR (Table 2). The results from the qPCR analysis for plant 3 are identical to the LAMP and conventional PCR results. However, the qPCR results for ZC-symptomatic plant 1 were negative for the tuber, leaf, and petiole samples, but positive for the stem sample. This demonstrates that qPCR was more sensitive than either qualitative PCR method in detecting ' $\mathrm{Ca}$. Liberibacter solanacearum' in field-grown potato plants.

Efficiency of LAMP in different isothermal reactors. LAMP was performed using the same reaction mix in different isothermal devices, including a thermocycler (normally used for variable temperature PCR), a heating block, an incubator, and a water bath. All devices were equally effective in LAMP amplification with a target temperature of $60^{\circ} \mathrm{C}$, demonstrating that a precision thermocycler is not required for LAMP.

\section{DISCUSSION}

Accurate pathogen detection of ' $\mathrm{Ca}$. Liberibacter' species in insect vectors and plant tissues is essential for the management of diseases such as ZC and HLB that are transmitted by psyllids. Although pathogen screening programs are already in place for the management of both diseases, these remain expensive (e.g., require thermal cycling equipment) and time consuming (require long amplification times and post-PCR analysis via gel electrophoresis or qPCR software). One of the most promising new methods for detection of ' $\mathrm{Ca}$. Liberibacter' is LAMP, which has been used successfully for medical diagnostics $(8,24)$ and to a limited extent for the rapid detection of plant-pathogenic bacteria within host specimens $(11,23,30)$. This report provides the nucleotide sequences for the LAMP primer set, LsoTX16SLAMP, and describes a complete protocol for LAMP detection of ' $\mathrm{Ca}$. Liberibacter solanacearum' in plant and insect hosts. We also discuss considerations for primer design, reaction optimization, and reductions in time required for pathogen detection.

The results using the LsoTX16SLAMP primer set and the optimized LAMP protocol demonstrated that the method effectively detected both ' $C a$. Liberibacter solanacearum' and ' $C a$. Liberibacter asiaticus' target sequences in plant DNA extracts. However, the primer set did not amplify target sequences of the closely related Rhizobiaceae members tested, i.e., Agrobacterium, Sinorhizobium, and Rhizobium species, indicating the primers may be used for the specific detection of both ' $\mathrm{Ca}$. Liberibacter' species.

On field samples, the LAMP method using the LsoTX16SLAMP primer set detected ' $C a$. Liberibacter solanacearum' with the same reliability as conventional PCR in bacterialiferous psyllids and in different tissues from ZC-symptomatic potato plants. However, both conventional PCR and LAMP failed to detect ' $\mathrm{Ca}$. Liberibacter solanacearum' in some samples from ZC-symptomatic plants. When these samples were tested by qPCR, it was determined that the bacterial titer in some of them (e.g., in a leaf from plant 3 and leaf, petiole and tuber samples from plant 1) were below the detection level even for qPCR. However, qPCR detected a low titer of ' $\mathrm{Ca}$. Liberibacter solanacearum' in the stem sample from ZC-symptomatic plant 1, which was determined to be ' $C a$. Liberibacter solanacearum'-negative via conventional PCR and LAMP for all tissues. Differences between conventional and qPCR in their ability to detect ' $\mathrm{Ca}$. Liberibacter solana- cearum' in different tissues of symptomatic plants has been observed previously and attributed to spatial and temporal variability in bacterial titer within the plant $(12,14,15)$. For example in experiments designed to look at the direction and rate of ' $\mathrm{Ca}$. Liberibacter solanacearum' movement in plants from a single leaf inoculation point, it was observed that bacteria spread from the infected leaf into the main stem within 7 days (12). Furthermore, populations reached titers sufficient for qPCR detection most reliably in immature leaves first and then older leaves, suggesting a source to sink pattern of movement; the study did not provide information on bacterial titers in tubers (12). These observations are consistent with qPCR detection of ' $\mathrm{Ca}$. Liberibacter solanacearum' in the stem samples of ZC-symptomatic plants 1 and 3, but not the mature leaves of either plant. Together these results indicate that LAMP is a very sensitive and robust method for ' $\mathrm{Ca}$. Liberibacter solanacearum' detection within insects and plants, but as with all qualitative PCR-based methods, it cannot detect the pathogen in all tissues of symptomatic plants when titers are low or bacteria are unequally distributed.

Development of a robust LAMP method requires careful design and testing of primers and optimization of the amplification reaction conditions, which are likely to be specific both to the primer set and the target gene sequence selected (10). However, by far the most challenging step is the design of effective primers. The LAMP turn-back primers, FIP and BIP, being required for stemloop formation, are the most crucial and we found that only HPLCpurified FIP and BIP primers provided reliable and reproducible amplification. As in the design of all PCR primers, LAMP primers require consideration of GC content, melting temperature, and the self-compatibility of the sequences and, for organisms such as ' $C a$. Liberibacter solanacearum' with a low genomic GC content, the selection of primer sequences with adequate GC content can be a critical determinant of LAMP success. In addition, because the formation of the loop structure is essential for LAMP amplification, further consideration of the free energy for hybridization at the 5'-end of the FIP and BIP primers and the 3 '-end of the F3, B3, FIP, BIP, LF, and LB primers as well as parameters related to secondary-structure formation are required. In this study, we were not successful in designing the LAMP primers without modifying them according to the UNAFold (16) and LASSO analysis (10). Equally important, we found that although LAMP as developed by Notomi et al. (20) requires only four primers (F3, B3, FIP, and BIP), reliable LAMP amplification could be achieved only by using the additional LF and LB primers, as described by Nagamine et al. (19). We recommend that all six of the LsoTX16SLAMP primers be used for LAMP detection of ' $\mathrm{Ca}$. Liberibacter solanacearum', as described in this study. Once the primer set is determined, it is important to perform assays to optimize the other reaction conditions, e.g., concentrations of Bst DNA polymerase enzyme, $\mathrm{MgSO}_{4}$, betaine; amplification temperature and time

TABLE 2. Comparison of polymerase chain reaction (PCR) detection of ' $\mathrm{Ca}$. Liberibacter solanacearum' using a conventional PCR primer pair (LsoTX $16 / 23 \mathrm{~F} / \mathrm{R}$ ), the newly developed loop-mediated isothermal amplification procedure (LAMP) primers (LsoTX16SLAMP), and a quantitative PCR (qPCR) primer pair (LsoF and HLBr) in assays of 10 zebra chip-symptomatic plants from a commercial field in Weslaco, TX

\begin{tabular}{lccc}
\hline $\begin{array}{l}\text { Plant parts } \\
\text { sampled }\end{array}$ & $\begin{array}{c}16 / 23 \mathrm{~F} / \mathrm{R} \\
\text { primer set }\end{array}$ & $\begin{array}{c}\text { 16S rDNA } \\
\text { LAMP primer set }\end{array}$ & $\begin{array}{c}\text { qPCR } \\
\text { primer set }\end{array}$ \\
\hline Tuber & $9 / 10(100.0 \%)^{\mathrm{a}}$ & $9 / 10(100.0 \%)^{\mathrm{a}}$ & $9 / 10(100.0 \%)^{\mathrm{a}}$ \\
Stem & $9 / 10(90.0 \%)$ & $9 / 10(90.0 \%)$ & $10 / 10(100.0 \%)$ \\
Petiole & $9 / 10(90.0 \%)$ & $9 / 10(90.0 \%)$ & $9 / 10(90.0 \%)$ \\
Leaf & $8 / 10(80.0 \%)$ & $8 / 10(80.0 \%)$ & $8 / 10(80.0 \%)$ \\
'Ca. Liberibacter & & & \\
$\quad$ solanacearum' & & & \\
detection total & $35 / 40(87.5 \%)$ & $35 / 40(87.5 \%)$ & $36 / 40(90.0 \%)$ \\
\hline
\end{tabular}

a Number of ' $\mathrm{Ca}$. Liberibacter solanacearum'-positive samples per total number (percentage). 
$(6,28,35)$. For LAMP using the LsoTX16SLAMP primer set, the $\mathrm{MgSO}_{4}$ concentration had the greatest effect on LAMP amplification; this is likely due to the effects of $\mathrm{Mg}^{2+}$ on DNA polymerase activity and primer annealing $(24,25)$.

The optimal amplification temperature and time conditions for LAMP using the LsoTX16SLAMP primer set were $60^{\circ} \mathrm{C}$ for $60 \mathrm{~min}$. However, fluctuations in the temperature during the amplification process (i.e., $60 \pm 3^{\circ} \mathrm{C}$ ) resulted in amounts of amplification products similar to precision controlled (e.g., thermocycler, $\pm 0.2^{\circ} \mathrm{C}$ ) amplification, indicating that a highly precise instrument is not required for LAMP amplification. In fact, our results demonstrated that a heating block, incubator, or water bath were equally capable of maintaining the temperature within this range and produced amounts of LAMP products equivalent to those produced in a thermocycler. In addition, LAMP amplification sufficient for ' $\mathrm{Ca}$. Liberibacter' detection was observed in as little as $45 \mathrm{~min}$, although amplification at $60 \mathrm{~min}$ was more reproducible.

These observations highlight two of the most significant advantages of the LAMP detection method, reliability and speed. Based on our work, an inexpensive dry bath such as the MyBlock Mini dry bath was a reliable and portable substitute for a thermocycler for the LAMP amplification. Furthermore, as observed in previous studies using LAMP (31), we found that all detection methods, e.g., direct, real-time monitoring of the turbidity of the reaction mixture or green color in the reaction vial, visualization of fluorescence under UV light (as green color or bright white in a gel imaging system), or via gel electrophoresis of the LAMP products were equally reproducible. Because observation of green color under normal light directly in the reaction tube requires no special equipment and positive and negative reactions are clearly discernible, we found this to be the preferred method for LAMP detection.

Although not the focus of this study, the LsoTX16SLAMP primer set and the reaction conditions employed for ' $\mathrm{Ca}$. Liberibacter solanacearum' detection was effective in detecting ' $\mathrm{Ca}$. Liberibacter asiaticus' in the limited number of citrus plant DNA extracts to which we had access. We also found that in our laboratory, this method was more robust than the previously published LAMP method for ' $\mathrm{Ca}$. Liberibacter asiaticus' detection employing sets of four LAMP primers designed for amplification of nucleotide sequences in the $16 \mathrm{~S}$ rDNA gene or the $n u s G$ $r p l K A J L-r p o B$ gene sequence (21). We speculate that our primer optimization using UNAFold and LASSO software and the inclusion of the additional loop primers contributed to the improved reliability of our method for ' $\mathrm{Ca}$. Liberibacter asiaticus' detection.

In summary, the LAMP method developed in this study has the potential to be extremely useful for the rapid detection of ' $\mathrm{Ca}$. Liberibacter solanacearum' and ' $\mathrm{Ca}$. Liberibacter asiaticus' and especially applicable in field laboratories without precise temperature controllers or electrophoresis and gel-imaging equipment. Ongoing work is focused on methods to adapt the LAMP method developed in this study for on-site testing of potato samples and psyllid vectors to facilitate real-time ' $\mathrm{Ca}$. Liberibacter solanacearum' detection and disease management decisions in the field.

\section{ACKNOWLEDGMENTS}

This work was supported by Texas A\&M AgriLife Research Project Number H-8832 (DCG). We thank C. Rush, G. Schuster, F. Workneh, and D. Henne for field samples; J. C. Miller, Jr., and D. Scheuring for potato seeds and technical advice on potato production; V. Vaughn, V. Alvarado, C. Tamborindeguy, and D. Odokonyero for technical advice, bacterialiferous psyllids, and supplies; and N. Wang, University of Florida \& Citrus Center at Lake Alfred, for ' $\mathrm{Ca}$. Liberibacter asiaticus' DNA samples.

\section{LITERATURE CITED}

1. Crosslin, J. M., Hamm, P. B., Eggers, J. E., Rondon, S. I., Sengoda, V. G., and Munyaneza, J. E. 2012. First report of zebra chip disease and "Candidatus Liberibacter solanacearum" on potatoes in Oregon and Washington State. Plant Dis. 96:452.

2. Crosslin, J. M., and Munyaneza, J. E. 2009. Evidence that the zebra chip disease and the putative causal agent can be maintained in potatoes by grafting and in vitro. Am. J. Potato Res. 86:183-187.

3. Crosslin, J. M., Olsen, N., and Nolte, P. 2012. First report of zebra chip disease and "Candidatus Liberibacter solanacearum" on potatoes in Idaho. Plant Dis. 96:453.

4. Doyle, J. J., and Doyle, J. L. 1990. Isolation of plant DNA from fresh tissue. Focus 12:13-15.

5. Hansen, A. K., Trumble, J. T., Stouthamer, R., and Paine, T. D. 2008. A new huanglongbing species, "Candidatus liberibacter psyllaurous," found to infect tomato and potato, is vectored by the psyllid Bactericera cockerelli (Sulc). Appl. Environ. Microbiol. 74:5862-5865.

6. Hara-Kudo, Y., Yoshino, M., Kojima, T., and Ikedo, M. 2005. Loopmediated isothermal amplification for the rapid detection of Salmonella. FEMS Microbiol. Lett. 253:155-161.

7. Hartung, J. S., Shao, J., and Kuykendall, L. D. 2011. Comparison of the ' $\mathrm{Ca}$. Liberibacter asiaticus' genome adapted for an intracellular lifestyle with other members of the Rhizobiales. Plos One 6(8):e23289.

8. Iwamoto, T., Sonobe, T., and Hayashi, K. 2003. Loop-mediated isothermal amplification for direct detection of Mycobacterium tuberculosis complex, M. avium, and M. intracellulare in sputum samples. J. Clin. Microbiol. 41:2616-2622.

9. Kim, J. S., Sagaram, U. S., Burns, J. K., Li, J. L., and Wang, N. 2009. Response of sweet orange (Citrus sinensis) to 'Candidatus Liberibacter asiaticus' infection: Microscopy and microarray analyses. Phytopathology 99:50-57.

10. Kimura, Y., de Hoon, M. J. L., Aoki, S., Ishizu, Y., Kawai, Y., Kogo, Y., Daub, C. O., Lezhava, A., Arner, E., and Hayashizaki, Y. 2011. Optimization of turn-back primers in isothermal amplification. Nucleic Acids Res. 39(9):1-8.

11. Kubota, R., Vine, B. G., Alvarez, A. M., and Jenkins, D. M. 2008. Detection of Ralstonia solanacearum by loop-mediated isothermal amplification. Phytopathology 98:1045-1051.

12. Levy, J., Ravindran, A., Gross, D., Tamborindeguy, C., and Pierson, E. 2011. Translocation of 'Candidatus Liberibacter solanacearum', the zebra chip pathogen, in potato and tomato. Phytopathology 101:1285-1291.

13. Li, W., Levy, L., and Hartung, J. S. 2009. Quantitative distribution of 'Candidatus Liberibacter asiaticus' in citrus plants with citrus huanglongbing. Phytopathology 99:139-144.

14. Li, W. B., Abad, J. A., French-Monar, R. D., Rascoe, J., Wen, A. M., Gudmestad, N. C., Secor, G. A., Lee, I. M., Duan, Y. P., and Levy, L. 2009. Multiplex real-time PCR for detection, identification and quantification of 'Candidatus Liberibacter solanacearum' in potato plants with zebra chip. J. Microbiol. Methods 78:59-65.

15. Li, W. B., Hartung, J. S., and Levy, L. 2006. Quantitative real-time PCR for detection and identification of Candidatus Liberibacter species associated with citrus huanglongbing. J. Microbiol. Methods 66:104-115.

16. Markham, N. R., and Zuker, M. 2008. UNAFold: Software for nucleic acid folding and hybridization. Methods Mol. Biol. 453:3-31.

17. Munyaneza, J. E., Goolsby, J. A., Crosslin, J. M., and Upton, J. E. 2007. Further evidence that zebra chip potato disease in the Lower Rio Grande Valley of Texas is associated with Bactericera cockerelli. Subtropical Plant Sci. 59:30-37.

18. Nachappa, P., Levy, J., Pierson, E., and Tamborindeguy, C. 2011. Diversity of endosymbionts in the potato psyllid, Bactericera cockerelli (Hemiptera: Triozidae), vector of zebra chip disease of potato. Curr. Microbiol. 62:1510-1520.

19. Nagamine, K., Hase, T., and Notomi, T. 2002. Accelerated reaction by loop-mediated isothermal amplification using loop primers. Mol. Cell Probe 16:223-229.

20. Notomi, T., Okayama, H., Masubuchi, H., Yonekawa, T., Watanabe, K., Amino, N., and Hase, T. 2000. Loop-mediated isothermal amplification of DNA. Nucleic Acids Res. 28(12):1-7.

21. Okuda, M., Matsumoto, M., Tanaka, Y., Subandiyah, S., and Iwanami, T. 2005. Characterization of the tufB-secE-nusG-rplKAJL-rpoB gene cluster of the citrus greening organism and detection by loop-mediated isothermal amplification. Plant Dis. 89:705-711.

22. Ravindran, A., Levy, J., Pierson, E., and Gross, D. C. 2011. Development of primers for improved PCR detection of the potato zebra chip pathogen, 'Candidatus Liberibacter solanacearum'. Plant Dis. 95:1542-1546.

23. Rigano, L. A., Marano, M. R., Castagnaro, A. P., Do Amaral, A. M., and Vojnov, A. A. 2010. Rapid and sensitive detection of citrus bacterial canker by loop-mediated isothermal amplification combined with simple visual evaluation methods. BMC Microbiol. 10(176):1-8. 
24. Saetiew, C., Limpaiboon, T., Jearanaikoon, P., Daduang, S., Pientong, C., Kerdsin, A., and Daduang, J. 2011. Rapid detection of the most common high-risk human papillomaviruses by loop-mediated isothermal amplification. J. Virol. Methods 178(1-2):22-30.

25. Saiki, R. K., Gelfand, D. H., Stoffel, S., Scharf, S. J., Higuchi, R., Horn, G. T., Mullis, K. B., and Erlich, H. A. 1988. Primer-directed enzymatic amplification of DNA with a thermostable DNA-polymerase. Science 239(4839):487-491.

26. Secor, G. A., Rivera, V. V., Abad, J. A., Lee, I. M., Clover, G. R. G., Liefting, L. W., Li, X., and De Boer, S. H. 2009. Association of 'Candidatus Liberibacter solanacearum' with Zebra Chip disease of potato established by graft and psyllid transmission, electron microscopy, and PCR. Plant Dis. 93:574-583.

27. Stackebrandt, E., and Goebel, B. M. 1994. Taxonomic note: A place for DNA-DNA reassociation and 16S rRNA sequence analysis in the present species definition in bacteriology. Int. J. Syst. Bacteriol. 44:846-849.

28. Sung, C. H., Chi, S. C., Huang, K. C., and Lu, J. K. 2010. Rapid detection of grouper Iridovirus by loop-mediated isothermal amplification. J. Mar. Sci. Tech.-Taiw. 18(4):568-573.

29. Tatineni, S., Sagaram, U. S., Gowda, S., Robertson, C. J., Dawson, W. O., Iwanami, T., and Wang, N. 2008. In planta distribution of 'Candidatus Liberibacter asiaticus' as revealed by polymerase chain reaction (PCR) and real-time PCR. Phytopathology 98:592-599.

30. Temple, T. N., and Johnson, K. B. 2011. Evaluation of loop-mediated isothermal amplification for rapid detection of Erwinia amylovora on pear and apple fruit flowers. Plant Dis. 95:423-430.

31. Tomita, N., Mori, Y., Kanda, H., and Notomi, T. 2008. Loop-mediated isothermal amplification (LAMP) of gene sequences and simple visual detection of products. Nat. Protoc. 3:877-882.

32. Tomlinson, J. A., Dickinson, M. J., and Boonham, N. 2010 Rapid detection of Phytophthora ramorum and $P$. kernoviae by twominute DNA extraction followed by isothermal amplification and amplicon detection by generic lateral flow device. Phytopathology 100:143-149.

33. Wen, A., Mallik, I., Alvarado, V. Y., Pasche, J. S., Wang, X., Li, W., Levy, L., Lin, H., Scholthof, H. B., Mirkov, T. E., Rush, C. M., and Gudmestad, N. C. 2009. Detection, distribution, and genetic variability of 'Candidatus Liberibacter' species associated with zebra complex disease of potato in North America. Plant Dis. 93:1102-1115.

34. Woese, C. R. 1987. Bacterial evolution. Microbiol. Rev. 51:221-271.

35. Yeh, H. Y., Shoemaker, C. A., and Klesius, P. H. 2005. Evaluation of a loop-mediated isothermal amplification method for rapid detection of channel catfish Ictalurus punctatus important bacterial pathogen Edwardsiella ictaluri. J. Microbiol. Methods 63:36-44. 